\title{
Nonlinear nonhomogeneous periodic problems
}

\author{
Giuseppina Barletta, Giuseppina D'Aguì \\ and Nikolaos S. Papageorgiou
}

\begin{abstract}
We consider a nonlinear periodic problem driven by a nonhomogeneous differential operator and a Carathéodory reaction. We show that it has at least three solutions, two of constant sign and the third nodal. In the particular case of the scalar $p$-Laplacian and with a parametric reaction of equidiffusive type, we show that three solutions with precise sign exist if the parameter $\lambda>\widehat{\lambda}_{1}(p)=$ the first nonzero eigenvalue of the periodic scalar Laplacian. Finally, in the semilinear case $(p=2)$, we show that there is a second nodal solution, for a total of four nontrivial solutions all with sign information.
\end{abstract}

Mathematics Subject Classification. 34B15, 34B18, 34C25, 58E05.

Keywords. Constant sign solutions, Extremal solutions, Nodal solutions, Nonlinear maximum principle, Critical groups.

\section{Introduction}

We study the following nonlinear periodic problem

$$
\left\{\begin{array}{l}
-\left(a\left(\left|u^{\prime}(t)\right|\right) u^{\prime}(t)\right)^{\prime}=f(t, u(t)) \quad \text { a.e. on } T=[0, b], \\
u(0)=u(b), u^{\prime}(0)=u^{\prime}(b) .
\end{array}\right.
$$

Here, the function $a(x)$, involved in the definition of the differential operator, is in general nonhomogeneous and incorporates the scalar $p$-Laplacian. The reaction $f(t, x)$ is a Carathéodory function (that is, for all $x \in \mathbb{R} t \rightarrow f(t, x)$ is measurable, while for a.a. $t \in T, x \rightarrow f(t, x)$ is continuous). We impose a growth condition on $f(t, \cdot)$ which makes the energy functional of the problem coercive. Using variational methods based on the critical point theory, we show that problem (1) has at least three nontrivial solutions and provide sign information for all of them (one is positive, the second is negative and the third is nodal (sign-changing)). As a particular case of our multiplicity theorem, we 
deal with the parametric periodic problem driven by the scalar $p$-Laplacian, with a reaction of the form

$$
f(t, x)=\lambda|x|^{p-2} x-h(t, x) \quad(1<p<\infty),
$$

where $\lambda>0$ is the parameter and $h(t, x)$ is a Carathéodory perturbation which is $(p-1)$-superlinear near to $\pm \infty$. We show that if $\hat{\lambda}_{1}(p)>0$ is the first nonzero eigenvalue of the periodic scalar $p$-Laplacian, then for all $\lambda>\hat{\lambda}_{1}(p)$ the problem has at least three nontrivial solutions again all with sign information. We mention that similar results were proved for semilinear (i.e., $p=2$ ) Dirichlet partial differential equations by Ambrosetti-Lupo [4], Ambrosetti-Mancini [5] and Struwe $[18,19]$. For $p$-Laplacian Dirichlet equations, we mention the work of Papageorgiou-Papageorgiou [16]. However, none of the aforementioned works produces a nodal solution. In the special case where $p=2$ (problem driven by the periodic scalar Laplacian), using tools from Morse theory (critical groups), we show that problem has a second nodal solution, for a total of four nontrivial solutions, all with sign information. Four nontrivial solutions, but without sign information for them, using techniques based on Morse theory, were also obtained by $\mathrm{Su}-\mathrm{Li}$ [21] for semilinear Dirichlet scalar equations. Closing, we mention the recent works of Aizicovici-Papageorgiou-Staicu [3] and KyritsiPapageorgiou [11] which deal with different classes of periodic problems driven by a nonhomogeneous differential operator.

\section{Mathematical background}

Let $X$ be a Banach space with $X^{*}$ its topological dual. By $\langle\cdot, \cdot\rangle$ we denote its duality brackets for the pair $\left(X^{*}, X\right)$. Given $\varphi \in C^{1}(X)$, we say that $\varphi$ satisfies the Palais-Smale condition (the $P S$-condition, for short), if the following is true:

"Every sequence $\left\{u_{n}\right\}_{n \geq 1} \subseteq X$ s.t. $\left\{\varphi\left(u_{n}\right)\right\}_{n \geq 1} \subseteq \mathbb{R}$ is bounded and

$$
\varphi^{\prime}\left(u_{n}\right) \rightarrow 0 \text { in } X^{*} \text { as } n \rightarrow \infty
$$

admits a strongly convergent subsequence."

This is a compactness-type condition on the functional $\varphi$, which compensates for the fact that the ambient space $X$ need not to be locally compact ( $X$ is in general infinite dimensional). This condition leads to a deformation theorem from which we can derive the mini-max theory of the critical values of $\varphi$. Prominent in this theory is the so-called mountain pass theorem due to Ambrosetti-Rabinowitz.

Theorem 2.1. If $X$ is a Banach space, $\varphi \in C^{1}(X)$ and satisfies the PS-condition, $u_{0}, u_{1} \in X,\left\|u_{1}-u_{0}\right\|>\rho>0$,

$$
\max \left\{\varphi\left(u_{0}\right), \varphi\left(u_{1}\right)\right\}<\inf \left[\varphi(u):\left\|u-u_{0}\right\|=\rho\right]=m_{\rho}, \text { and }
$$

$c=\inf _{\gamma \in \Gamma} \max _{0 \leq t \leq 1} \varphi(\gamma(t))$, where $\Gamma=\left\{\gamma \in C([0,1], X): \gamma(0)=u_{0}\right.$, $\left.\gamma(1)=u_{1}\right\}$, then $c>m_{\rho}$ and $c$ is a critical value of $\varphi$. 
In the analysis of problem (1) we will use the Sobolev space

$$
W:=W_{p e r}^{1, p}(0, b)=\left\{u \in W^{1, p}(0, b): u(0)=u(b)\right\}
$$

and the Banach space

$$
\hat{C}^{1}(T)=C^{1}(T) \cap W \quad(\text { recall } T=[0, b]) .
$$

The Sobolev space $W^{1, p}(0, b)$ is embedded continuously (in fact compactly) in $C(T)$ and so the evaluations of $u(\cdot)$ at $t=0$ and at $t=b$ in the definition of $W$ make sense. The space $\hat{C}^{1}(T)$ is an ordered Banach space with positive cone

$$
\hat{C}_{+}=\left\{u \in \hat{C}^{1}(T): u(t) \geq 0 \text { for all } t \in T\right\},
$$

which has a nonempty interior given by

$$
\operatorname{int} \hat{C}_{+}=\left\{u \in \hat{C}_{+}: u(t)>0 \text { for all } t \in T\right\} .
$$

In what follows, by $\|\cdot\|$ we denote the norm on the Sobolev space $W$, that is

$$
\|u\|=\left[\|u\|_{p}^{p}+\left\|u^{\prime}\right\|_{p}^{p}\right]^{\frac{1}{p}} \text { for all } u \in W .
$$

The hypotheses on the map $a(\cdot)$ involved in the differential operator are the following.

$H(a): a:(0, \infty) \rightarrow(0, \infty)$ is a $C^{1}$-function such that

(i) $x \rightarrow a(x) x$ is strictly increasing on $(0, \infty), a(x) x \rightarrow 0^{+}$as $x \rightarrow 0^{+}$and

$$
\lim _{x \rightarrow 0^{+}} \frac{a^{\prime}(x) x}{a(x)}=c>-1 ;
$$

(ii) there exist $\hat{c}>0$ and $1<p<\infty$ s.t.

$$
|a(|x|) x| \leq \hat{c}\left(1+|x|^{p-1}\right) \text { for all } x \in \mathbb{R}
$$

(iii) there exists $c_{0}>0$ s.t.

$$
a(|x|) x^{2} \geq c_{0}|x|^{p} \text { for all } x \in \mathbb{R} ;
$$

(iv) if $G_{0}(x)=\int_{0}^{x} a(s) s d s$ for all $x \geq 0$, then

$$
p G_{0}(x)-a(x) x^{2} \geq 0 \text { for all } x \geq 0
$$

and there exists $q \in(1, p]$ s.t.

$$
x \rightarrow G_{0}\left(x^{\frac{1}{q}}\right) \text { is convex on }(0,+\infty) \text { and } \lim _{x \rightarrow 0^{+}} \frac{q G_{0}(x)}{x^{q}}=\tilde{c}>0 .
$$

Remark 2.1. Evidently $G_{0}(\cdot)$ is strictly convex and strictly increasing on $(0, \infty)$. We set

$$
G(x)=G_{0}(|x|) \text { for all } x \in \mathbb{R} .
$$

Then $G$ is strictly convex and for $x \neq 0$ we have

$$
G^{\prime}(x)=G_{0}^{\prime}(|x|)=a(|x|)|x| \cdot \frac{x}{|x|}=a(|x|) x .
$$

So, $G(\cdot)$ is the primitive of the function $x \rightarrow a(|x|) x, x \in \mathbb{R}$. Since $G(0)=0$ and $G(\cdot)$ is convex, we have

$$
G(x) \leq a(|x|) x^{2} \text { for all } x \in \mathbb{R} .
$$


Then from (2) and hypotheses $H(a)(i i),(i i i)$, we obtain

$$
\frac{c_{0}}{p}|x|^{p} \leq G(x) \leq c_{1}\left(1+|x|^{p}\right) \text { for some } c_{1}>0 \text { and all } x \in \mathbb{R} .
$$

Examples. The following functions satisfy hypotheses $H(a)$ above:

$$
\begin{aligned}
& a_{1}(|x|) x=|x|^{p-2} x \text { with } 1<p<+\infty, \\
& a_{2}(|x|) x=|x|^{p-2} x+|x|^{q-2} x \text { with } 1<q<p<+\infty, \\
& a_{3}(|x|) x=\left(1+x^{2}\right)^{\frac{p-2}{2}} x \text { with } 1<p<+\infty, \\
& a_{4}(|x|) x=|x|^{p-2} x+\frac{|x|^{p-2} x}{1+|x|^{p}} \text { with } 1<p<+\infty .
\end{aligned}
$$

Corresponding potential (primitive) functions are:

$$
\begin{aligned}
G_{1}(x) & =\frac{1}{p}|x|^{p}, \\
G_{2}(x) & =\frac{1}{p} x^{p}+\frac{1}{q}|x|^{q}, \\
G_{3}(x) & =\frac{1}{p}\left[\left(1+x^{2}\right)^{\frac{p}{2}}-1\right], \\
G_{4}(x) & =\frac{1}{p}|x|^{p}+\frac{1}{p} \ln \left(1+|x|^{p}\right) .
\end{aligned}
$$

We mention that $a_{1}(x) x$ corresponds to the scalar $p$-Laplacian, $a_{2}(x) x$ corresponds to the $(p, q)$-differential operator and $a_{3}(x)$ corresponds to the generalized scalar $p$-mean curvature differential operator.

Let $V: W \rightarrow W^{*}$ be the nonlinear map defined by

$$
\langle V(u), y\rangle=\int_{0}^{b} a\left(\left|u^{\prime}\right|\right) u^{\prime} y^{\prime} d t \text { for all } u, y \in W .
$$

The next result can be found in Motreanu-Motreanu-Papageorgiou [13] (p. 405).

Proposition 2.1. If hypotheses $H(a)$ hold and $V: W \rightarrow W^{*}$ is the nonlinear map defined by (4), then $V$ is of type $(S)_{+}$, that is if $u_{n} \rightarrow u$ in $W$ and

$$
\limsup _{n \rightarrow+\infty}\left\langle V\left(u_{n}\right), u_{n}-u\right\rangle \leq 0 \text {, }
$$

then $u_{n} \rightarrow u$ in $W$.

Suppose that $f_{0}: T \times \mathbb{R} \rightarrow \mathbb{R}$ is a Carathéodory function s.t.

$$
\left|f_{0}(t, x)\right| \leq \alpha(t)\left(1+|x|^{r-1}\right) \text { for a.a. } t \in T, \text { all } x \in \mathbb{R}
$$

with $\alpha \in L^{1}(T)_{+}, 1<r<+\infty$. We set $F_{0}(t, x)=\int_{0}^{x} f_{0}(t, s) d s$ and consider the $C^{1}$-functional $\Psi_{0}: W \rightarrow \mathbb{R}$ defined by

$$
\Psi_{0}(u)=\int_{0}^{b} G\left(u^{\prime}(t)\right) d t-\int_{0}^{b} F_{0}(t, u(t)) d t \text { for all } u \in W .
$$

The next result relates smooth and Sobolev local minimizers for $\Psi_{0}$ and can be found in Aizicovici-Papageorgiou-Staicu [3]. We mention that the first such 
result was proved by Brezis-Niremberg [7] for functionals defined on $H_{0}^{1}(\Omega)$ $\left(\Omega \subseteq \mathbb{R}^{N}\right.$ a bounded domain).

Proposition 2.2. If hypotheses $H(a)$ hold and $u_{0} \in W$ is a local $\hat{C}^{1}(T)$-minimizer of $\Psi_{0}$, that is there exists $\rho_{0}>0$ s.t.

$$
\Psi_{0}\left(u_{0}\right) \leq \Psi_{0}\left(u_{0}+h\right) \text { for all } h \in \hat{C}^{1}(T) \text {, with }\|h\|_{\hat{C}^{1}(T)} \leq \rho_{0},
$$

then $u_{0} \in \hat{C}^{1}(T)$ and it is a local $W$-minimizer of $\Psi_{0}$, that is there exists $\rho_{1}>0$ s.t.

$$
\Psi_{0}\left(u_{0}\right) \leq \Psi_{0}\left(u_{0}+h\right) \quad \text { for all } h \in W, \text { with }\|h\| \leq \rho_{1} .
$$

If as before, $X$ is a Banach space and $\varphi \in C^{1}(X), c \in \mathbb{R}$, then we introduce the following sets

$\varphi^{c}=\{u \in X: \varphi(u) \leq c\}, K_{\varphi}=\left\{u \in X: \varphi^{\prime}(u)=0\right\}$ and $K_{\varphi}^{c}=\left\{u \in K_{\varphi}: \varphi(u)=c\right\}$.

If $Y_{2} \subseteq Y_{1} \subseteq X$, then for every integer $k \geq 0$, by $H_{k}\left(Y_{1}, Y_{2}\right)$ we denote the $k^{\text {th }}$-relative singular homology group with integer coefficients. The critical groups of $\varphi$ at an isolated critical point $u_{0} \in X$ with $\varphi\left(u_{0}\right)=c$ (that is $\left.u_{0} \in K_{\varphi}^{c}\right)$, are defined by

$$
C_{k}\left(\varphi, u_{0}\right)=H_{k}\left(\varphi^{c} \cap \mathcal{U}, \varphi^{c} \cap \mathcal{U} \backslash\left\{u_{0}\right\}\right), \quad \text { for all } k \geq 0 .
$$

Here $\mathcal{U}$ is a neighborhood of $u_{0}$ such that $K_{\varphi} \cap \varphi^{c} \cap \mathcal{U}=\left\{u_{0}\right\}$. The excision property of singular homology, implies that the above definition is independent of the particular choice of neighborhood $\mathcal{U}$.

Suppose that $\varphi$ satisfies the $P S$-condition and $-\infty<\inf \varphi\left(K_{\varphi}\right)$. Let $c<\inf \varphi\left(K_{\varphi}\right)$. The critical groups of $\varphi$ at infinity are defined by

$$
C_{k}(\varphi, \infty)=H_{k}\left(X, \varphi^{c}\right) \text { for all } k \geq 0 .
$$

The second deformation theorem (see, for example Gasinski-Papageorgiou [10], p. 628), implies that the above definition is independent of the particular choice of the level $c<\inf \varphi\left(K_{\varphi}\right)$.

Suppose that $K_{\varphi}$ is finite. We introduce the following quantities:

$$
\begin{aligned}
& M(t, u)=\sum_{k \geq 0} \operatorname{rank} C_{k}(\varphi, u) t^{k} \text { for all } t \in \mathbb{R}, \text { all } u \in K_{\varphi}, \\
& P(t, \infty)=\sum_{k \geq 0} \operatorname{rank} C_{k}(\varphi, \infty) t^{k} \text { for all } t \in \mathbb{R} .
\end{aligned}
$$

The Morse relation says that

$$
\sum_{u \in K_{\varphi}} M(t, u)=P(t, \infty)+(1+t) Q(t),
$$

where $Q(t)=\sum_{k \geq 0} \beta_{k} t^{k}$ is a formal series in $t \in \mathbb{R}$, with nonnegative integer coefficients.

Finally, we fix our notations in this paper. Given $x \in \mathbb{R}$ we set $x^{ \pm}=$ $\max \{ \pm x, 0\}$. Then for every $u \in W$, we define $u^{ \pm}(\cdot)=u(\cdot)^{ \pm}$. We have

$$
u=u^{+}-u^{-},|u|=u^{+}+u^{-} \text {and } u^{ \pm},|u| \in W .
$$


Given a measurable function $h: T \times \mathbb{R} \rightarrow \mathbb{R}$ (for example a Carathéodory function), we set

$$
N_{h}(u)(\cdot)=h(\cdot, u(\cdot)) \quad \text { for all } u \in W
$$

(the Nemytskii or superposition operator corresponding to $h$ ). By $|\cdot|_{1}$ we denote the Lebesgue measure on $\mathbb{R}$.

\section{Solutions of constant sign}

In this section, we produce two nontrivial solutions of constant sign (one positive and the other negative) for problem (1). The hypotheses on the reaction $f(t, x)$ are the following (here $q \in(1, p]$ is as in hypothesis $H(a)(i v)$ ).

$H_{1}: f: T \times \mathbb{R} \rightarrow \mathbb{R}$ is a Carathéodory function s.t. $f(t, 0)=0$ for a.a. $t \in T$ and

(i) for every $\rho>0$ there exists $\alpha_{\rho} \in L^{\infty}(T)$ s.t.

$$
|f(t, x)| \leq \alpha_{\rho}(t) \text { for a.a. } t \in T \text {, all }|x| \leq \rho ;
$$

(ii) $\lim _{x \rightarrow \pm \infty} \frac{f(t, x)}{|x|^{p-2} x}=-\infty$ uniformly for a.a. $t \in T$;

(iii) there exists $\eta \in L^{\infty}(T), \eta(t) \geq 0$ a.e. on $T, \eta \neq 0$ s.t.

$$
\eta(t) \leq \liminf _{x \rightarrow 0} \frac{f(t, x)}{|x|^{q-2} x} \text { uniformly for a.a. } t \in T
$$

(iv) for every $\rho>0$ there exists $\xi_{\rho}>0$ s.t.

$f(t,-\rho)-\xi_{\rho} \rho^{p-1} \leq f(t,-x)-\xi_{\rho}|x|^{p-1} \leq 0 \leq f(t, x)+\xi_{\rho}|x|^{p-1} \leq f(t, \rho)+\xi_{\rho} \rho^{p-1}$

for all $x \in[0, \rho]$, for all $t \in T$.

Remark 3.1. Hypothesis $H_{1}(i v)$ is weaker than the more common: for every $\rho>0$ there exists $\xi_{\rho}>0$ s.t. $x \rightarrow f(t, x)+\xi_{\rho}|x|^{p-2} x$ is not decreasing for all $x \in[-\rho, \rho]$, for all $t \in T$. In fact if the latter holds true then $H_{1}(i v)$ is satisfied, but the converse is no longer true. Take for instance $p=3, q=2$ and

$$
f(x)= \begin{cases}-x^{3}-8 & \text { if } x<-2 \\ \sqrt{-x-1}-1 & \text { if }-2 \leq x<-1 \\ x & \text { if }-1 \leq x \leq 1 \\ -\sqrt{x-1}+1 & \text { if } 1 \leq x<2 \\ -x^{3}+8 & \text { if } x>2\end{cases}
$$

Then $f$ satisfies hypotheses $H_{1}$ but if we take $\rho \in(1,2)$ then for all $\xi>0$ the function $x \rightarrow f(t, x)+\xi|x| x$ has at least a point of minimum $x_{\rho} \in[-\rho, \rho]$, so it is not non decreasing.

We stress that $f(t, \cdot)$ does not satisfy any global growth condition. Only that $-f(t, \cdot)$ near $\pm \infty$ growths faster then $|x|^{p-2} x((p-1)$-superlinearity, see $\left.H_{1}(i i)\right)$. This condition implies that we can find $M_{1}>0$ s.t.

$$
f(t, x) x \leq-1<0 \text { for a.a } t \in T \text {, all }|x| \geq M_{1} .
$$

Based on this observation, we choose $\xi>M_{1}$ and we have

$$
0=V(\xi) \geq N_{f}(\xi) \text { in } W^{*}
$$




$$
0=V(-\xi) \leq N_{f}(-\xi) \text { in } W^{*} .
$$

We consider the following ordered intervals in $W^{1, p}(0, b)$

$$
\begin{gathered}
{[0, \xi]=\left\{u \in W^{1, p}(0, b): 0 \leq u(t) \leq \xi \text { for all } t \in T\right\}} \\
{[-\xi, 0]=\left\{u \in W^{1, p}(0, b):-\xi \leq u(t) \leq 0 \text { for all } t \in T\right\} .}
\end{gathered}
$$

Then we introduce the following truncations-perturbations of $f(t, \cdot)$

$$
\begin{aligned}
k_{+}(t, x) & = \begin{cases}0 & \text { if } x<0 \\
f(t, x)+x^{p-1} & \text { if } 0 \leq x \leq \xi \\
f(t, \xi)+\xi^{p-1} & \text { if } \xi<x\end{cases} \\
\text { and } \quad k_{-}(t, x) & = \begin{cases}f(t,-\xi)+|\xi|^{p-2} \xi & \text { if } x<-\xi \\
f(t, x)+|x|^{p-2} x & \text { if }-\xi \leq x \leq 0 \\
0 & \text { if } 0<x\end{cases}
\end{aligned}
$$

Both are Carathéodory functions. We set $K_{ \pm}(t, x)=\int_{0}^{x} k_{ \pm}(t, s) d s$ and consider the $C^{1}$-functionals $\hat{\varphi}_{ \pm}: W \rightarrow \mathbb{R}$ defined by

$$
\hat{\varphi}_{ \pm}(u)=\int_{0}^{b} G\left(u^{\prime}(t)\right) d t+\frac{1}{p}\|u\|_{p}^{p}-\int_{0}^{b} K_{ \pm}(t, u(t)) d t \text { for all } u \in W .
$$

Also let $\varphi: W \rightarrow \mathbb{R}$ be the energy functional for problem (1) defined by

$$
\varphi(u)=\int_{0}^{b} G\left(u^{\prime}(t)\right) d t-\int_{0}^{b} F(t, u(t)) d t \text { for all } u \in W,
$$

where $F(t, x)=\int_{0}^{x} f(t, s) d s$. Evidently $\varphi \in C^{1}(W)$.

Proposition 3.1. If hypotheses $H(a)$ and $H_{1}$ hold, then problem (1) has at least two nontrivial constant sign solutions

$$
u_{0} \in \operatorname{int}_{\hat{C}^{1}(T)}[0, \xi], v_{0} \in-\operatorname{int}_{\hat{C}^{1}(T)}[-\xi, 0]
$$

both local minimizers of the energy functional $\varphi$.

Proof. First we produce the positive solution.

From (9) it is clear that the functional $\hat{\varphi}_{+}$is coercive. Moreover, using the Sobolev embedding theorem, we see that $\hat{\varphi}_{+}$is sequentially weakly lower semicontinuous. So, by the Weierstrass theorem, we can find $u_{0} \in W$ s.t.

$$
\hat{\varphi}_{+}\left(u_{0}\right)=\min \left[\hat{\varphi}_{+}(u): u \in W\right] .
$$

Note that hypothesis $H_{1}($ iii $)$ implies that given $\varepsilon>0$, we can find $\delta=\delta(\varepsilon)>0$ s.t.

$$
F(t, x) \geq \frac{1}{q}(\eta(t)-\varepsilon)|x|^{q} \text { for a.a. } t \in T, \text { all }|x| \leq \delta .
$$

So, if the constant $\beta \in[0, \delta]$, then [see (9) and (12)]

$$
\hat{\varphi}_{+}(\beta)=-\int_{0}^{b} F(t, \beta) d t \leq \frac{\beta^{q}}{q}\left[-\int_{0}^{b} \eta(t) d t+\varepsilon b\right] .
$$

Choosing $\varepsilon \in\left(0, \frac{1}{b} \int_{0}^{b} \eta(t) d t\right)$ [see hypothesis $\left.H_{1}(i i i)\right]$, we see that

$$
\hat{\varphi}_{+}(\beta)<0 \text {, so, using also (11), we have }
$$




$$
\hat{\varphi}_{+}\left(u_{0}\right)<0=\hat{\varphi}_{+}(0) \text {, hence } u_{0} \neq 0 \text {. }
$$

From (11) we have $\hat{\varphi}_{+}^{\prime}\left(u_{0}\right)=0$, that is

$$
V\left(u_{0}\right)+\left|u_{0}\right|^{p-2} u_{0}=N_{k_{+}}\left(u_{0}\right) .
$$

On (13) we act with $-u_{0}^{-} \in W$. Then, from (9)

$$
\int_{0}^{b} a\left(\left|\left(u_{0}^{-}\right)^{\prime}\right|\right)\left(\left(u_{0}^{-}\right)^{\prime}\right)^{2} d t+\left\|u_{0}^{-}\right\|_{p}^{p}=0
$$

so, using hypothesis $H(a)(i i i)$, we deduce

$$
c_{0}\left\|\left(u_{0}^{-}\right)^{\prime}\right\|_{p}^{p}+\left\|u_{0}^{-}\right\|_{p}^{p} \leq 0, \text { so }\left\|u_{0}^{-}\right\|=0,
$$

hence $u_{0} \geq 0$ and $u_{0} \neq 0$.

Also on (13) we act with $\left(u_{0}-\xi\right)^{+} \in W$. Then, from (9), (7) and the fact that $V(\xi)=0$

$$
\begin{array}{r}
\left\langle V\left(u_{0}\right),\left(u_{0}-\xi\right)^{+}\right\rangle+\int_{0}^{b} u_{0}^{p-1}\left(u_{0}-\xi\right)^{+} d t=\int_{0}^{b}\left[f(t, \xi)+\xi^{p-1}\right]\left(u_{0}-\xi\right)^{+} d t \\
\leq \int_{0}^{b} \xi^{p-1}\left(u_{0}-\xi\right)^{+} d t=\left\langle V(\xi),\left(u_{0}-\xi\right)^{+}\right\rangle+\int_{0}^{b} \xi^{p-1}\left(u_{0}-\xi\right)^{+} d t
\end{array}
$$

so,

$$
\left\langle V\left(u_{0}\right)-V(\xi),\left(u_{0}-\xi\right)^{+}\right\rangle+\int_{0}^{b}\left(u_{0}^{p-1}-\xi^{p-1}\right)\left(u_{0}-\xi\right)^{+} d t \leq 0,
$$

hence $\left|\left\{u_{0}>\xi\right\}\right|_{1}=0$ and so $u_{0} \leq \xi$.

Therefore we have proved that $u_{0} \in[0, \xi] \backslash\{0\}$. Then (see (9)) (13) becomes

$$
V\left(u_{0}\right)=N_{f}\left(u_{0}\right) \text {, hence }-\left(a\left(\left|u_{0}^{\prime}(t)\right|\right) u_{0}^{\prime}(t)\right)^{\prime}=f\left(t, u_{0}(t)\right) \text { a.e. on } T,
$$

and from [3] we infer

$$
u(0)=u(b), u^{\prime}(0)=u^{\prime}(b),
$$

so $u_{0}$ is a nontrivial positive solution of (1) belonging to $\hat{C}_{+} \backslash\{0\}$. Let $\rho=$ $\left\|u_{0}\right\|_{\infty}$ and let $\xi_{\rho}>0$ be as in $H_{1}(i v)$. Then

$$
-\left(a\left(\left|u_{0}^{\prime}(t)\right|\right) u_{0}^{\prime}(t)\right)^{\prime}+\xi_{\rho} u_{0}(t)^{p-1}=f\left(t, u_{0}(t)\right)+\xi_{\rho} u_{0}(t)^{p-1} \geq 0 \text { a.e. on } T,
$$

so

$$
\left(a\left(\left|u_{0}^{\prime}(t)\right|\right) u_{0}^{\prime}(t)\right)^{\prime} \leq \xi_{\rho} u_{0}(t)^{p-1} \text { a.e. on } T .
$$

From the strong maximum principle of Pucci-Serrin [17] (p. 111), we have $u_{0}(t)>0$ for all $t \in(0, b)$. Invoking the boundary point theorem of PucciSerrin [17] (p. 120), we conclude that

$$
u_{0} \in \operatorname{int} \hat{C}_{+} .
$$

Let $\delta^{\prime}>0$ and set $u_{\delta^{\prime}}(t)=u_{0}(t)+\delta^{\prime}$. Then $u_{\delta^{\prime}} \in \operatorname{int} \hat{C}_{+}$. Let $\rho=\xi$ and let $\xi_{\rho}>0$ be as in $H_{1}(i v)$. Since $u_{0}(t) \leq \xi$ for all $t \in T$ we can use $H_{1}(i v)$, while 
our choice of $\xi$ guarantees (see $(6))$ that $f(t, \xi)<0$ for a.a. $t \in T$. Hence, we can find $c\left(\delta^{\prime}\right) \rightarrow 0^{+}$as $\delta^{\prime} \rightarrow 0^{+}$such that

$$
\begin{gathered}
-\left(a\left(\left|u_{\delta^{\prime}}^{\prime}\right|\right) u_{\delta^{\prime}}^{\prime}\right)^{\prime}+\xi_{\rho} u_{\delta^{\prime}}^{p-1} \leq-\left(a\left(\left|u_{0}^{\prime}\right|\right) u_{0}^{\prime}\right)^{\prime}+\xi_{\rho} u_{0}^{p-1}+c\left(\delta^{\prime}\right)= \\
f\left(t, u_{0}\right)+\xi_{\rho} \xi^{p-1}+c\left(\delta^{\prime}\right) \leq \xi_{\rho} \xi^{p-1} \text { for } \delta^{\prime} \text { small enough, hence } \\
\xi-u_{0} \in \operatorname{int} \hat{C}_{+} .
\end{gathered}
$$

From (14) and (15) we conclude that

$$
u_{0} \in \operatorname{int}_{\hat{C}^{1}(T)}[0, \xi] .
$$

Note that (9) yields $\hat{\varphi}_{+\mid[0, \xi]}=\varphi_{\mid[0, \xi]}$, so (16) guarantees that $u_{0}$ is a local $C^{1}(T)$-minimizer of $\varphi$, hence (see Proposition 2.2) also a local $W$-minimizer of $\varphi$. In a similar fashion, working with $\hat{\varphi}_{-}$and using this time (10), we produce a second nontrivial constant sign solution $v_{0} \in \operatorname{int}_{\hat{C}^{1}(T)}[-\xi, 0]$ which is a local minimizer of the functional $\varphi$.

In fact, we can produce extremal nontrivial constant sign solutions, that is the smallest positive solution and the biggest negative solution. We will need them in the next section in order to generate a nodal (sign changing) solution.

To this end, note that by virtue of hypotheses $H_{1}(i),($ iii $)$, given $\varepsilon>0$ and $r>p$, we can find $c_{2}=c_{2}(\varepsilon, r)>0$ such that

$$
f(t, x) \geq(\eta(t)-\varepsilon)|x|^{q}-c_{2}|x|^{r} \text { for a.a. } t \in T, \text { all }|x| \leq \xi .
$$

This unilateral growth estimate on $f(t, \cdot)$, leads the following auxiliary nonlinear periodic problem

$$
\left\{\begin{array}{l}
-\left(a\left(\left|u^{\prime}(t)\right|\right) u^{\prime}(t)\right)^{\prime}=(\eta(t)-\varepsilon)|u(t)|^{q-2} u(t)-c_{2}|u(t)|^{r-2} u(t) \text { a.e. on } T, \\
u(0)=u(b), u^{\prime}(0)=u^{\prime}(b) .
\end{array}\right.
$$

Proposition 3.2. If hypotheses $H(a)$ hold, then problem (18) has a positive solution $\tilde{u} \in \operatorname{int} \hat{C}_{+}$and since $(18)$ is odd, $\tilde{v}=-\tilde{u} \in-\operatorname{int} \hat{C}_{+}$is the unique negative solution of (18).

Proof. First we establish the existence of a nontrivial positive solution for problem (18). So, let $\Psi_{+}: W \rightarrow \mathbb{R}$ be the $C^{1}$-functional defined by

$\Psi_{+}(u)=\int_{0}^{b} G\left(u^{\prime}\right) d t+\frac{1}{p}\|u\|_{p}^{p}+\frac{c_{2}}{r}\left\|u^{+}\right\|_{r}^{r}-\frac{1}{q} \int_{0}^{b}(\eta(t)-\varepsilon)\left(u^{+}(t)\right)^{q} d t-\frac{1}{p}\left\|u^{+}\right\|_{p}^{p}$

for all $u \in W$.

Put $c_{3}=\|\eta\|_{\infty}$. Because $p<r$, we can find $c_{4}>0$ such that

$$
\begin{aligned}
\Psi_{+}(u) & \geq \frac{c_{0}}{p}\|u\|_{p}^{p}+\frac{c_{2}}{r}\left\|u^{+}\right\|_{r}^{r}-\frac{c_{3}}{q}\left\|u^{+}\right\|_{q}^{q}-\frac{1}{p}\left\|u^{+}\right\|_{p}^{p} \\
& \geq \frac{c_{0}}{p}\|u\|_{p}^{p}+\left[\frac{c_{2}}{r}\left\|u^{+}\right\|_{r}^{r-p}-c_{4}\right]\left\|u^{+}\right\|_{r}^{p}-\frac{c_{3}}{q}\left\|u^{+}\right\|_{q}^{q}
\end{aligned}
$$


Since $q \leq p<r$, from (19) we infer that $\Psi_{+}$is coercive. Also, via the Sobolev embedding theorem, we see that $\Psi_{+}$is sequentially weakly lower semicontinuous. So, by the Weierstrass theorem, we can find $\tilde{u} \in W$ s.t.

$$
\Psi_{+}(\tilde{u})=\min \left[\Psi_{+}(u): u \in W\right] .
$$

Let $\theta \in(0, \infty)$. We have

$$
\Psi_{+}(\theta)=\frac{c_{2} \theta^{r}}{r} b-\frac{\theta^{q}}{q} \int_{0}^{b}(\eta(t)-\varepsilon) d t=\frac{c_{2} \theta^{r}}{r} b-\frac{\theta^{q}}{q}\left[\int_{0}^{b} \eta(t) d t-\varepsilon b\right] .
$$

Choosing $\varepsilon \in\left(0, \frac{1}{b} \int_{0}^{b} \eta(t) d t\right)$ and recalling that $q<r$, we see that for $\theta \in(0,1)$ small we will have

$$
\Psi_{+}(\theta)<0, \text { and so, from }(20) \Psi_{+}(\tilde{u})<0=\Psi_{+}(0) \text {, hence } \tilde{u} \neq 0 .
$$

From $(20)$ we know $\Psi_{+}^{\prime}(\tilde{u})=0$, that is

$$
V(\tilde{u})+|\tilde{u}|^{p-2} \tilde{u}=(\eta(t)-\varepsilon)\left(\tilde{u}^{+}\right)^{q-1}-c_{2}\left(\tilde{u}^{+}\right)^{r-1}+\left(\tilde{u}^{+}\right)^{p-1} .
$$

On $(21)$ we act with $-\tilde{u}^{-} \in W$. Then

$$
\left\langle V(\tilde{u}),-\tilde{u}^{-}\right\rangle+\left\|\tilde{u}^{-}\right\|_{p}^{p}=0,
$$

so, using hypothesis $H(a)(i i i)$ we deduce

$$
c_{0}\left\|\left(\tilde{u}^{-}\right)^{\prime}\right\|_{p}^{p}+\left\|\tilde{u}^{-}\right\|_{p}^{p} \leq 0, \text { so } \tilde{u} \geq 0, \tilde{u} \neq 0 .
$$

So (21) becomes

$$
\begin{aligned}
V(\tilde{u}) & =(\eta(t)-\varepsilon) \tilde{u}^{q-1}-c_{2} \tilde{u}^{r-1}, \text { that is } \\
-\left(a\left(\left|\tilde{u}^{\prime}(t)\right|\right) \tilde{u}^{\prime}(t)\right)^{\prime} & =(\eta(t)-\varepsilon) \tilde{u}(t)^{q-1}-c_{2} \tilde{u}(t)^{r-1} \text { a.e. on } T,
\end{aligned}
$$

and from [3] we infer

$$
\tilde{u}(0)=\tilde{u}(b), \tilde{u}^{\prime}(0)=\tilde{u}^{\prime}(b)
$$

so $\tilde{u} \in \hat{C}_{+} \backslash\{0\}$ solves the auxiliary problem (18).

We have

$$
\left(a\left(\left|\tilde{u}^{\prime}(t)\right|\right) \tilde{u}^{\prime}(t)\right)^{\prime} \leq c_{2}\|\tilde{u}\|_{\infty}^{r-p} \tilde{u}(t)^{p} \text { a.e. in } T,
$$

so, by using the results of Pucci-Serrin [17], $\tilde{u} \in \operatorname{int} \hat{C}_{+}$. Next we show that in fact $\tilde{u} \in \operatorname{int} \hat{C}_{+}$is the unique positive solution of (18). To this end, let $\gamma_{+}: L^{q}(T) \rightarrow \overline{\mathbb{R}}=\mathbb{R} \cup\{+\infty\}$ be the integral functional defined by

$$
\gamma_{+}(u)= \begin{cases}\int_{0}^{b} G\left(\left(u^{1 / q}\right)^{\prime}\right) d t & \text { if } u^{1 / q} \in W, u \geq 0 \\ +\infty & \text { otherwise. }\end{cases}
$$

Let $u_{1}, u_{2} \in \operatorname{dom} \gamma_{+}=\left\{u \in L^{q}(T): \gamma_{+}(u)<+\infty\right\}$. We set $v_{1}=u_{1}^{1 / q}$, $v_{2}=u_{2}^{1 / q}$ and for $s \in[0,1]$ we define

$$
v=\left((1-s) u_{1}+s u_{2}\right)^{1 / q} \text {. }
$$

Using Lemma 1 of Diaz-Saa [8], we have

$$
\left|v^{\prime}(t)\right| \leq\left[(1-s)\left|v_{1}^{\prime}(t)\right|^{q}+s\left|v_{2}^{\prime}(t)\right|^{q}\right]^{1 / q} \text { for a.a. } t \in T \text {. }
$$


Using the monotonicity of $G_{0}$ and hypothesis $H(a)(i v)$ we get

$$
\begin{aligned}
G_{0}\left(\left|v^{\prime}(t)\right|\right) \leq & G_{0}\left((1-s)\left|v_{1}^{\prime}(t)\right|^{q}+s\left|v_{2}^{\prime}(t)\right|^{q}\right)^{1 / q} \leq(1-s) G_{0}\left(\left|v_{1}^{\prime}(t)\right|\right) \\
& +s G_{0}\left(\left|v_{2}^{\prime}(t)\right|\right) \text { for a.a. } t \in T .
\end{aligned}
$$

Therefore

$$
G\left(v^{\prime}(t)\right) \leq(1-s) G\left(v_{1}^{\prime}(t)\right)+s G\left(v_{2}^{\prime}(t)\right) \text { for a.a. } t \in T,
$$

so $\gamma_{+}$is convex. Also, via Fatou's lemma, we have that $\gamma_{+}$is lower semicontinuous.

Let $u, v$ be two positive solutions of problem (18). From the first part of the proof we know that $u, v \in \operatorname{int} \hat{C}_{+}$. Then for any $h \in \hat{C}^{1}(T)$ and for $|\lambda| \leq 1$ small, we have

$$
u^{q}+\lambda h \in \operatorname{dom} \gamma_{+} \text {and } v^{q}+\lambda h \in \operatorname{dom} \gamma_{+} .
$$

So, $\gamma_{+}$is Gâteaux differentiable at $u^{q}, v^{q}$ in the direction $h$ and using the chain rule and the density of $\hat{C}^{1}(T)$ in $W$, we obtain

$$
\begin{aligned}
& \gamma_{+}^{\prime}\left(u^{q}\right)(h)=\frac{1}{q} \int_{0}^{b} \frac{-\left(a\left(\left|u^{\prime}\right|\right) u^{\prime}\right)^{\prime}}{u^{q-1}} h d t \text { and } \\
& \gamma_{+}^{\prime}\left(v^{q}\right)(h)=\frac{1}{q} \int_{0}^{b} \frac{-\left(a\left(\left|v^{\prime}\right|\right) v^{\prime}\right)^{\prime}}{v^{q-1}} h d t \text { for all } h \in W .
\end{aligned}
$$

The convexity of $\gamma_{+}$, implies the monotonicity of $\gamma_{+}^{\prime}$. Hence

$$
\begin{aligned}
0 & \leq \frac{1}{q} \int_{0}^{b}\left[\frac{(\eta(t)-\varepsilon) u^{q-1}-c_{2} u^{r-1}}{u^{q-1}}-\frac{(\eta(t)-\varepsilon) v^{q-1}-c_{2} v^{r-1}}{v^{q-1}}\right]\left(u^{q}-v^{q}\right) d t \\
& =\frac{1}{q} \int_{0}^{b} c_{2}\left[v^{r-q}-u^{r-q}\right]\left(u^{q}-v^{q}\right) d t \leq 0,
\end{aligned}
$$

so $u=v$. This proves the uniqueness of $\tilde{u} \in \operatorname{int} \hat{C}_{+}$. Evidently $\tilde{v}=-\tilde{u} \in$ $-\operatorname{int} \hat{C}_{+}$is the unique negative solution of (18).

We introduce the following sets:

$$
\begin{aligned}
& S_{+}=\{u \in W: u \in[0, \xi] \text { and it is a positive solution of }(1)\} \\
& S_{-}=\{v \in W: v \in[-\xi, 0] \text { and it is a negative solution of }(1)\} .
\end{aligned}
$$

From Proposition 3.1 and its proof, we know that

$$
\begin{aligned}
& S_{+} \neq \emptyset \text { and } S_{+} \subseteq[0, \xi] \cap \operatorname{int} \hat{C}_{+} \\
& S_{-} \neq \emptyset \text { and } S_{-} \subseteq[-\xi, 0] \cap\left(-\operatorname{int} \hat{C}_{+}\right) .
\end{aligned}
$$

In addition, from Aizicovici-Papageorgiou-Staicu [3] (see also FilippakisKristaly-Papageorgiou [9]), we have that $S_{+}$is downward directed (that is, if $u_{1}, u_{2} \in S_{+}$, then there exists $u \in S_{+}$s.t. $u \leq u_{1}, u \leq u_{2}$ ), while $S_{-}$is upward directed (that is, if $v_{1}, v_{2} \in S_{-}$, then there exists $v \in S_{-}$s.t. $v_{1} \leq v, v_{2} \leq v$ ).

Proposition 3.3. If hypotheses $H(a)$ and $H_{1}$ hold, then problem (1) has a smallest positive solutions $u_{*} \in \operatorname{int} \hat{C}_{+}$and a biggest negative solution $v_{*} \in-\operatorname{int} \hat{C}_{+}$. 
Proof. Let $\tilde{u} \in \operatorname{int} \hat{C}_{+}$be the unique positive solution of the auxiliary problem (18).

Claim: $\tilde{u} \leq u$ for all $u \in S_{+}$.

function

Let $u \in S_{+}, \varepsilon \in\left(0, \frac{\|\eta\|_{1}}{b}\right)$ and consider the following Carathéodory

$$
h_{+}(t, x)= \begin{cases}0 & \text { if } x<0 \\ (\eta(t)-\varepsilon) x^{q-1}-c_{2} x^{r-2}+x^{p-1} & \text { if } 0 \leq x \leq u(t) \\ (\eta(t)-\varepsilon) u(t)^{q-1}-c_{2} u(t)^{r-2}+u(t)^{p-1} & \text { if } u(t)<x\end{cases}
$$

We set $H_{+}(t, x)=\int_{0}^{x} h_{+}(t, s) d s$ and consider the $C^{1}$-functional $\beta_{+}$: $W \rightarrow \mathbb{R}$ defined by

$$
\beta_{+}(u)=\int_{0}^{b} G\left(u^{\prime}(t)\right) d t+\frac{1}{p}\|u\|_{p}^{p}-\int_{0}^{b} H_{+}(t, u(t)) \text { for all } u \in W .
$$

From (3) and (16), it is clear that $\beta_{+}$is coercive. Also, it is sequentially weakly lower semicontinuous. So, we can find $\tilde{u}_{0} \in W$ s.t.

$$
\beta_{+}\left(\tilde{u}_{0}\right)=\min \left[\beta_{+}(u): u \in W\right] .
$$

Since $u \in \operatorname{int} \hat{C}_{+}$, we can choose $\theta \in\left(0, \min _{T} u\right)$. Using $(22)$ we obtain

$$
\beta_{+}(\theta)=-\frac{\theta^{q}}{q} \int_{0}^{b}(\eta(t)-\varepsilon) d t+\frac{\theta^{r}}{r} c_{2}
$$

Since $q<r$, choosing $\theta$ even smaller if necessary, from (24), we see that

$$
\beta_{+}(\theta)<0, \text { so } \beta_{+}\left(\tilde{u}_{0}\right)<0=\beta_{+}(0) \text {, hence } \tilde{u}_{0} \neq 0 \text {. }
$$

From (23), we have $\beta_{+}^{\prime}\left(\tilde{u}_{0}\right)=0$, that is

$$
V\left(\tilde{u}_{0}\right)+\left|\tilde{u}_{0}\right|^{p-2} \tilde{u}_{0}=N_{h_{+}}\left(\tilde{u}_{0}\right) .
$$

On (25) first we act with $-\tilde{u}_{0}^{-} \in W$. Using $H(a)(i i i)$ and (22), we obtain

$$
c_{0}\left\|\left(\tilde{u}_{0}^{-}\right)^{\prime}\right\|_{p}^{p}+\left\|\tilde{u}_{0}^{-}\right\|_{p}^{p} \leq 0 \text { so } \tilde{u}_{0} \geq 0, \tilde{u}_{0} \neq 0 .
$$

Also on $(25)$, se act with $\left(\tilde{u}_{0}-u\right)^{+} \in W$. Then, taking into account (22)

$$
\begin{aligned}
& \left\langle V\left(\tilde{u}_{0}\right),\left(\tilde{u}_{0}-u\right)^{+}\right\rangle+\int_{0}^{b} \tilde{u}_{0}^{p-1}\left(\tilde{u}_{0}-u\right)^{+} d t=\int_{0}^{b} h_{+}\left(t, \tilde{u}_{0}\right)\left(\tilde{u}_{0}-u\right)^{+} d t \\
& =\int_{0}^{b}\left[(\eta(t)-\varepsilon) u^{q-1}-c_{2} u^{r-1}+u^{p+1}\right]\left(\tilde{u}_{0}-u\right)^{+} d t \\
& =\left\langle V(u),\left(\tilde{u}_{0}-u\right)^{+}\right\rangle+\int_{0}^{b} u^{p-1}\left(\tilde{u}_{0}-u\right)^{+} d t
\end{aligned}
$$

SO,

$$
\left\langle V\left(\tilde{u}_{0}\right)-V(u),\left(\tilde{u}_{0}-u\right)^{+}\right\rangle+\int_{0}^{b}\left(\tilde{u}_{0}^{p-1}-u^{p-1}\right)\left(\tilde{u}_{0}-u\right)^{+} d t=0
$$

from which we obtain $\left|\left\{\tilde{u}_{0}>u\right\}\right|_{1}=0$, hence $\tilde{u}_{0} \leq u$.

So, we have proved that $\tilde{u}_{0} \in[0, u] \backslash\{0\}$. Therefore (25) becomes

$$
V\left(\tilde{u}_{0}\right)=(\eta(t)-\varepsilon) \tilde{u}_{0}^{q-1}-c_{2} \tilde{u}_{0}^{r-1}
$$


that is

$$
\begin{aligned}
-\left(a\left(\left|\tilde{u}_{0}^{\prime}(t)\right|\right) \tilde{u}_{0}^{\prime}(t)\right)^{\prime} & =(\eta(t)-\varepsilon) \tilde{u}_{0}^{q-1}(t)-c_{2} \tilde{u}_{0}^{r-1}(t) \text { a.e. on } T, \\
\tilde{u}_{0}(0) & =\tilde{u}_{0}(b), \tilde{u}_{0}^{\prime}(0)=\tilde{u}_{0}^{\prime}(b) .
\end{aligned}
$$

Finally, taking into account Proposition 3.2 , we have $\tilde{u}_{0}=\tilde{u} \in \operatorname{int} \hat{C}_{+}$, so $\tilde{u} \leq u$ for all $u \in S_{+}$and this proves the claim.

For the set $S_{+}$, we know that we can find a sequence $\left\{u_{n}\right\} \subseteq S_{+}$(which can be taken to be decreasing since $S_{+}$is downward directed) s.t. inf $S_{+}=$ $\inf _{n \geq 1} u_{n}$. We have

$$
V\left(u_{n}\right)=N_{f}\left(u_{n}\right) \text { and } \tilde{u} \leq u_{n} \leq \xi \text { for all } n \geq 1 .
$$

From (26) and hypotheses $H(a)(i i i)$ and $H_{1}(i)$ it follows that $\left\{u_{n}\right\}_{n \geq 1} \subseteq W$ is bounded. So we may assume that

$$
u_{n} \rightarrow u_{*} \text { in } W \text { and } u_{n} \rightarrow u_{*} \text { in } C(T) .
$$

On the Eq. (26), we act with $u_{n}-u_{*} \in W$, pass to the limit as $n \rightarrow \infty$ and use (27). Then

$$
\lim _{n \rightarrow \infty}\left\langle V\left(u_{n}\right), u_{n}-u_{*}\right\rangle=0,
$$

so by Proposition 2.1, $u_{n} \rightarrow u_{*} \in W$, and $V\left(u_{*}\right)=N_{f}\left(u_{*}\right), \tilde{u} \leq u_{*} \leq \xi$, then $u_{*}=\inf S_{+}$and so $u_{*} \leq u$ for all $u \in S_{+}$.

Similarly, working with $S_{-}$we obtain $\sup S_{-}=v_{*} \in S_{-}$and so $v \leq v_{*}$ for all $v \in S_{-}$.

\section{Nodal solutions}

In this section, using the extremal constant sign solutions $u_{*} \in \operatorname{int} \hat{C}_{+}$and $v_{*} \in-\operatorname{int} \hat{C}_{+}$produced in Proposition 3.3, we will generate a nodal solution. We consider the order interval $\left[v_{*}, u_{*}\right]=\left\{u \in W^{1, p}(0, b): v_{*}(t) \leq u(t) \leq\right.$ $u_{*}(t)$ for all $\left.t \in T\right\}$. Using Theorem 2.1, we show that problem (1) admits a nontrivial solution $y_{0} \in\left[v_{*}, u_{*}\right]$ distinct from $v_{*}$ and $u_{*}$. Evidently the extremality of $v_{*}$ and $u_{*}$ guarantees that $y_{0}$ is nodal. For this strategy to work, we need to strengthen the condition on the reaction $f(t, \cdot)$ near 0 . Consider the following eigenvalue problem

$$
\left\{\begin{array}{l}
-\left(\left(\left|u^{\prime}(t)\right|\right)^{q-2} u^{\prime}(t)\right)^{\prime}=\hat{\lambda}|u(t)|^{q-2} u(t) \text { a.e. on } T \\
u(0)=u(b), u^{\prime}(0)=u^{\prime}(b) .
\end{array}\right.
$$

Here $1<q \leq p$ is as in hypothesis $H(a)(i v)$. We say that $\hat{\lambda} \in \mathbb{R}$ is an eigenvalue of the periodic scalar $q$-Laplacian, if problem (28) admits a nontrivial solution $\hat{u}$ known as an eigenfunction corresponding to $\hat{\lambda}$. We know that $\hat{u} \in \hat{C}^{1}(T) \backslash\{0\}$. The periodic scalar $q$-Laplacian admits a sequence of eigenvalues $\left\{\hat{\lambda}_{k}(q)\right\}_{k \geq 0}$ s.t.

$$
0=\hat{\lambda}_{0}(q)<\hat{\lambda}_{1}(q)<\cdots<\hat{\lambda}_{k}(q) \rightarrow+\infty \text { as } k \rightarrow \infty
$$


In fact $\hat{\lambda}_{k}(q)=\left(\frac{2 k \pi_{q}}{b}\right)^{q}$ with $\pi_{q}=\frac{2 \pi(q-1)^{\frac{1}{q}}}{q \sin \left(\frac{\pi}{q}\right)}$. Note that if $q=2$, then $\pi_{q}=\pi$ and we recover the well known sequence of eigenvalues of the periodic scalar Laplacian, given by $\left\{\hat{\lambda}_{k}(2)=\left(\frac{2 k \pi}{b}\right)^{2}\right\}_{k>0}$. Every eigenfunction $\hat{u} \in C^{1}(T)$ of $(28)$ satisfies $\hat{u}(t) \neq 0$ a.e. on $T$ and in fact $\hat{u}(\cdot)$ has a finite number of zeros. The first eigenvalue $\hat{\lambda}_{0}(q)=0$ is simple with constant eigenfunctions. It is the only eigenvalue with eigenfunctions of constant sign. All the other eigenvalues have nodal eigenfunctions. Let $\hat{u}_{0}(q)$ be the $L^{q}-$ normalized positive eigenfunction corresponding to $\hat{\lambda}_{0}(q)=0$. We have

$$
\hat{u}_{0}(q)=\frac{1}{b^{1 / q}} \in \operatorname{int} \hat{C}_{+} .
$$

Let $\partial B_{1}^{L^{q}}=\left\{u \in L^{q}(T):\|u\|_{q}=1\right\}$ and let $M=W \cap \partial B_{1}^{L^{q}}$. From AizicoviciPapageorgiou-Staicu [2], we have the following minimax characterization of $\hat{\lambda}_{1}(q)>0$.

Proposition 4.1. $\hat{\lambda}_{1}(q)=\inf _{\hat{\gamma} \in \hat{\Gamma}} \max _{-1 \leq s \leq 1}\left\|\frac{d}{d t} \hat{\gamma}(s)\right\|_{q}^{q}$, where $\hat{\Gamma}=\left\{\hat{\gamma} \in C([-1,1], M): \hat{\gamma}(-1)=-\hat{u}_{0}(q), \hat{\gamma}(1)=\hat{u}_{0}(q)\right\}$.

The new stronger hypotheses on the reaction $f(t, x)$ are the following: $H_{2}: f: T \times \mathbb{R} \rightarrow \mathbb{R}$ is a Carathéodory function s.t. $f(t, 0)=0$ for a.a. $t \in$ $T$, hypotheses $H_{2}(i),(i i),(i v)$ are the same as the corresponding hypotheses $H_{1}(i),(i i)(i v)$ and

(iii) for $q \in(1, p]$ and $\tilde{c}>0$ as in hypothesis $H(a)(i v)$, there exists $\eta>0$ s.t.

$$
\tilde{c} \hat{\lambda}_{1}(q)<\eta \leq \liminf _{x \rightarrow 0} \frac{f(t, x)}{|x|^{q-2} x} \text { uniformly for a.a. } t \in T \text {. }
$$

Let $u_{*}$ and $v_{*}$ be the two extremal constant sign solutions produced in Proposition 3.3. We introduce the following Carathéodory truncation-perturbations of $f(t, \cdot)$ :

$h(t, x)= \begin{cases}f\left(t, v_{*}(t)\right)+\left|v_{*}(t)\right|^{p-2} v_{*}(t) & \text { if } x<v_{*}(t) \\ f(t, x)+|x|^{p-2} x & \text { if } v_{*}(t) \leq u_{*}(t), h_{ \pm}(t, x)=h\left(t, \pm x^{ \pm}\right) . \\ f\left(t, u_{*}(t)\right)+u_{*}(t)^{p-1} & \text { if } u_{*}(t) \leq x\end{cases}$

We set $H(t, x)=\int_{0}^{x} h(t, s) d s, H_{ \pm}(t, x)=\int_{0}^{x} h_{ \pm}(t, s) d s$ and consider the $C^{1}$ functionals $\sigma, \sigma_{ \pm}: W \rightarrow \mathbb{R}$ defined by

$$
\begin{gathered}
\sigma(u)=\int_{0}^{b} G\left(u^{\prime}(t)\right) d t+\frac{1}{p}\|u\|_{p}^{p}-\int_{0}^{b} H(t, u(t)) d t \text { for all } u \in W . \\
\sigma_{ \pm}(u)=\int_{0}^{b} G\left(u^{\prime}(t)\right) d t+\frac{1}{p}\|u\|_{p}^{p}-\int_{0}^{b} H_{ \pm}(t, u(t)) d t \text { for all } u \in W .
\end{gathered}
$$

Reasoning as in the proof of Proposition 3.3 we can check that

$$
K_{\sigma} \subseteq\left[v_{*}, u_{*}\right], \quad K_{\sigma_{+}} \subseteq\left[0, u_{*}\right], K_{\sigma_{-}} \subseteq\left[v_{*}, 0\right] .
$$


The extremality of $u_{*}$ and $v_{*}$ implies that

$$
K_{\sigma} \subseteq\left[v_{*}, u_{*}\right], \quad K_{\sigma_{+}}=\left\{0, u_{*}\right\}, K_{\sigma_{-}}=\left\{v_{*}, 0\right\} .
$$

Also we have:

Proposition 4.2. If hypotheses $H(a)$ and $H_{2}$ hold, then $u_{*}$ and $v_{*}$ are both local minimizers of $\sigma$.

Proof. It is clear from (29) that $\sigma_{+}$is coercive and sequentially weakly lower semicontinuous. So, we can find $\hat{u}_{*} \in W$ s.t.

$$
\sigma_{+}\left(\hat{u}_{*}\right)=\min \left[\sigma_{+}(u): u \in W\right] .
$$

As before, using hypothesis $H_{2}(i i i)$, we obtain $\sigma_{+}\left(\hat{u}_{*}\right)<0=\sigma_{+}(0)$, hence $\hat{u}_{*} \neq 0$ and by $(30)$ and $(31) \hat{u}_{*}=u_{*}$.

Since $u_{*} \in \operatorname{int} \hat{C}_{+}$and $\sigma_{+\mid \hat{C}_{+}}=\sigma_{\mid \hat{C}_{+}}$, we see that $u_{*}$ is a local $\hat{C}^{1}(T)-$ minimizer of $\sigma$, hence by virtue of Proposition 2.2 it is also a local $W$-minimizer of $\sigma$. Similarly for $v_{*} \in-\operatorname{int} \hat{C}_{+}$using this time the functional $\sigma_{-}$.

Now, we are ready to generate a nodal solution for problem (1).

Proposition 4.3. If hypotheses $H(a)$ and $H_{2}$ hold, then problem (1) admits a nodal solution $y_{0} \in\left[v_{*}, u_{*}\right] \cap \hat{C}^{1}(T)$.

Proof. Without loss of generality we may assume that $\sigma\left(v_{*}\right) \leq \sigma\left(u_{*}\right)$ (the analysis is similar if the opposite inequality holds). Because of Proposition 4.2 (see also the proof of Proposition 29 in [1]), we can find $\rho \in(0,1)$ small s.t.

$$
\sigma\left(v_{*}\right) \leq \sigma\left(u_{*}\right)<\inf \left[\sigma(u):\left\|u-u_{*}\right\|=\rho\right]=m_{\rho}, \quad\left\|v_{*}-u_{*}\right\|>\rho .
$$

$\sigma$ is coercive (see (29)), so it satisfies the $P S$-condition. This fact and (32) permit the use of Theorem 2.1. Hence, we can find $y_{0} \in W$ s.t.

$$
y_{0} \in K_{\sigma} \text { and } m_{\rho} \leq \sigma\left(y_{0}\right)=\inf _{\gamma \in \Gamma} \max _{0 \leq s \leq 1} \sigma(\gamma(s)),
$$

where

$$
\Gamma=\left\{\gamma \in C([0,1], W): \gamma(0)=v_{*}, \gamma(1)=u_{*}\right\} .
$$

From (30), (32) and (33) it follows that $y_{0} \in\left[v_{*}, u_{*}\right]$ and $y_{0} \notin\left\{u_{*}, v_{*}\right\}$. So, if $y_{0} \neq 0$, then it will be the desired nodal solution. According to (33), in order to show the nontriviality of $y_{0}$, it suffices to produce a path $\gamma_{*} \in \Gamma$ s.t. $\sigma_{\mid \gamma_{*}}<0$. Let us construct such a path. Consider the $C^{1}$-manifolds $M=W \cap \partial B_{1}^{L^{q}}$ furnished with the relative $W$-topology and $M_{c}=M \cap \hat{C}^{1}(T)$ furnished with the relative $\hat{C}^{1}(T)$-topology. We take also the following sets of paths

$$
\begin{aligned}
\hat{\Gamma} & =\left\{\hat{\gamma} \in C([-1,1], M): \hat{\gamma}(-1)=-\hat{u}_{0}(q), \hat{\gamma}(1)=\hat{u}_{0}(q)\right\} \text { and } \\
\hat{\Gamma}_{c} & =\left\{\hat{\gamma} \in C\left([-1,1], M_{c}\right): \hat{\gamma}(-1)=-\hat{u}_{0}(q), \hat{\gamma}(1)=\hat{u}_{0}(q)\right\} .
\end{aligned}
$$

Claim: $\hat{\Gamma}_{c}$ is dense in $\hat{\Gamma}$ for the $C([-1,1], M)$-topology.

Let $\hat{\gamma} \in \hat{\Gamma}$ and $\varepsilon \in(0,1)$. We consider the multifunction $L_{\varepsilon}:[-1,1] \rightarrow$ $2^{\hat{C}^{1}(T)} \backslash\{0\}$ defined by

$$
L_{\varepsilon}(s)=\left\{u \in \hat{C}^{1}(T):\|u(s)-\hat{\gamma}(s)\|<\varepsilon\right\} \text { for all } s \in(-1,1)
$$




$$
L_{\varepsilon}(-1)=-\hat{u}_{0}(q), L_{\varepsilon}(1)=\hat{u}_{0}(q) .
$$

$L_{\varepsilon}$ has convex and open values for all $s \in(-1,1)$, while $L_{\varepsilon}(-1)$ and $L_{\varepsilon}(1)$ are singletons. Therefore $L_{\varepsilon}$ has values in the family $\mathfrak{D}\left(\hat{C}^{1}(T)\right)$ of Michael [12]. Also, the continuity of $\hat{\gamma}$ implies that $L_{\varepsilon}$ is lower semicontinuous (see KyritsiPapageorgiou [15], (p.458)). So, we can apply Theorem 3.1"' of Michael [12] and produce a continuous map $\hat{\gamma}_{\varepsilon}$ s.t. $\hat{\gamma}_{\varepsilon}(s) \in L_{\varepsilon}(s)$ for all $s \in[-1,1]$. Let $\varepsilon_{n}=\frac{1}{n}, n \geq 1$ and $\left\{\hat{\gamma}_{n}=\hat{\gamma}_{\varepsilon_{n}}\right\}_{n \geq 1} \subseteq C\left([-1,1], C^{1}(T)\right)$ s.t.

$$
\left\|\hat{\gamma}_{n}(s)-\hat{\gamma}(s)\right\|<\frac{1}{n}, \text { for all } s \in[-1,1], \hat{\gamma}_{n}( \pm 1)= \pm \hat{u}_{0}(q) .
$$

Since $\|\hat{\gamma}(s)\|_{q}=1$ for all $s \in[-1,1]$, we may assume that $\left\|\hat{\gamma}_{n}(s)\right\| \neq 0$ for all $s \in(-1,1)$. Therefore, we can define

$$
\tilde{\gamma}_{n}(s)=\frac{\hat{\gamma}_{n}(s)}{\left\|\hat{\gamma}_{n}(s)\right\|_{q}} \text { for all } s \in[-1,1]
$$

satisfying

$$
\tilde{\gamma}_{n} \in C\left([-1,1], M_{c}\right), \quad \tilde{\gamma}_{n}( \pm 1)= \pm \hat{u}_{0}(q) .
$$

Moreover, for every $s \in[-1,1]$ and every $n \geq 1$, due to (34) we have:

$$
\begin{aligned}
\left\|\tilde{\gamma}_{n}(s)-\hat{\gamma}(s)\right\| & \leq\left\|\tilde{\gamma}_{n}(s)-\hat{\gamma}_{n}(s)\right\|+\left\|\hat{\gamma}_{n}(s)-\hat{\gamma}(s)\right\| \\
& <\frac{\left|1-\left\|\hat{\gamma}_{n}(s)\right\|_{q}\right|}{\left\|\hat{\gamma}_{n}(s)\right\|_{q}}\left\|\hat{\gamma}_{n}(s)\right\|+\frac{1}{n} .
\end{aligned}
$$

Further, taking into account that $\|\hat{\gamma}(s)\|_{q}=1$ and (34), we can find $c_{1}>0$ s.t. for all $n \geq 1$ one has

$$
\begin{aligned}
\max _{-1 \leq s \leq 1}\left|1-\left\|\hat{\gamma}_{n}(s)\right\|_{q}\right| & =\max _{-1 \leq s \leq 1}\left|\|\hat{\gamma}(s)\|_{q}-\left\|\hat{\gamma}_{n}(s)\right\|_{q}\right| \\
& \leq \max _{-1 \leq s \leq 1}\left\|\hat{\gamma}(s)-\hat{\gamma}_{n}(s)\right\|_{q} \leq c_{1}\left\|\hat{\gamma}(s)-\hat{\gamma}_{n}(s)\right\|<\frac{c_{1}}{n}
\end{aligned}
$$

If we insert the last inequality in (35), then we infer

$$
\lim _{n \rightarrow+\infty} \max _{-1 \leq s \leq 1}\left\|\tilde{\gamma}_{n}(s)-\hat{\gamma}(s)\right\|=0
$$

and this proves the Claim.

Hypotheses $H(a)(i v)$ and $H_{2}($ iii $)$ imply that given $\varepsilon>0$, we can find $\delta=\delta(\varepsilon)>0$ s.t.

$\frac{\eta-\varepsilon}{q}|x|^{q} \leq F(t, x)$ for a.a. $t \in T$, all $|x| \leq \delta, G_{0}(t) \leq \frac{(\tilde{c}+\varepsilon) t^{q}}{q}$ for all $t \in[0, \delta]$.

The Claim proved above and Proposition 4.1 imply that, given $\theta>0$, we can find $\tilde{\gamma} \in \hat{\Gamma}_{c}$ and $\lambda=\lambda(\tilde{\gamma})$ s.t.

$$
\max _{-1 \leq s \leq 1}\left\|\frac{d}{d t} \tilde{\gamma}(s)\right\|_{q}^{q} \leq \hat{\lambda}_{1}(q)+\theta
$$


and (see Lemma 3.3 of Filippakis-Kristàly-Papageorgiou [9])

$$
\lambda \tilde{\gamma}(s) \in\left[v_{*}, u_{*}\right], \lambda|\tilde{\gamma}(s)(t)|, \lambda\left|\frac{d}{d t} \tilde{\gamma}(s)(t)\right| \leq \delta \text { for all } s \in[-1,1], \quad \text { all } \quad t \in T .
$$

Then for all $s \in[-1,1]$, if we use $(29),(37),(36),(38)$ and recall that $\|\tilde{\gamma}(s)\|_{q}=$ 1 , then we have

$$
\begin{array}{r}
\sigma(\lambda \tilde{\gamma}(s))=\int_{0}^{b} G\left(\lambda \tilde{\gamma}(s)^{\prime}(t)\right) d t-\int_{0}^{b} F(t, \tilde{\gamma}(s)(t)) d t \leq \frac{\lambda^{q}}{q}(\tilde{c}+\varepsilon)\left\|\frac{d}{d t} \tilde{\gamma}(s)\right\|_{q}^{q}-\frac{\lambda^{q}}{q}(\eta-\varepsilon) \\
\leq \frac{\lambda^{q}}{q}(\tilde{c}+\varepsilon)\left(\hat{\lambda}_{1}(q)+\theta\right)-\frac{\lambda^{q}}{q}(\eta-\varepsilon)=\frac{\lambda^{q}}{q}\left[\tilde{c} \hat{\lambda}_{1}(q)-\eta+\varepsilon\left(\hat{\lambda}_{1}(q)+\theta+1\right)+\tilde{c} \theta\right] .
\end{array}
$$

Recall $\eta>\tilde{c} \hat{\lambda}_{1}(q)$. So, choosing $\varepsilon, \theta$ small, from (39) we see that

$$
\sigma(\lambda \tilde{\gamma}(s))<0 \text { for all } s \in[-1,1] .
$$

Hence, $\tilde{\gamma}_{\lambda}=\lambda \tilde{\gamma}$ is a continuous path in $W$ which connects $-\lambda \hat{u}_{0}(q)$ with $\lambda \hat{u}_{0}(q)$ and

$$
\sigma_{\mid \tilde{\gamma}_{\lambda}}<0
$$

From the proof of Proposition 3.3 we know that $\alpha=\sigma_{+}\left(u_{*}\right)<0=\sigma_{+}(0)$, so by virtue of the second deformation theorem (see, for example, GasinskiPapageorgiou [10] (p. 628)), we can find $h:[0,1] \times\left(\sigma_{+}^{0} \backslash K_{\sigma_{+}}^{0}\right) \rightarrow \sigma_{+}^{0}$ a continuous map s.t.

$$
\begin{gathered}
h(0, u)=u \text { for all } u \in \sigma_{+}^{0} \backslash K_{\sigma_{+}}^{0}, \\
h\left(1, \sigma_{+}^{0} \backslash K_{\sigma_{+}}^{0}\right) \subseteq \sigma_{+}^{\alpha}, \\
\sigma_{+}\left(h\left(\tau^{\prime}, u\right)\right) \leq \sigma_{+}(h(\tau, u)) \text { for all } \tau^{\prime}, \tau \in[0,1], \tau \leq \tau^{\prime}, u \in \sigma_{+}^{0} \backslash K_{\sigma_{+}}^{0} .
\end{gathered}
$$

From (30) we know that $K_{\sigma_{+}}=\left\{0, u_{*}\right\}$. Therefore $\sigma_{+}^{\alpha}=\left\{u_{*}\right\}$. Since $\sigma_{+\mid \hat{C}_{+}}=$ $\sigma_{\mid \hat{C}_{+}}$, from (40) we obtain

$$
\sigma_{+}\left(\lambda \hat{u}_{0}(q)\right)=\sigma\left(\lambda \hat{u}_{0}(q)\right)=\sigma(\tilde{\gamma}(1))<0
$$

and $\lambda \hat{u}_{0}(q) \subseteq \sigma_{+}^{0} \backslash K_{\sigma_{+}}^{0}=\sigma_{+}^{0} \backslash\{0\}$. Therefore, we can define the continuous path

$$
\tilde{\gamma}^{+}(\tau)=h\left(\tau, \lambda \hat{u}_{0}(q)\right)^{+} \text {for all } \tau \in[0,1]
$$

for which we have

$$
\begin{gathered}
\tilde{\gamma}_{\lambda}^{+}(0)=\lambda \hat{u}_{0}(q), \tilde{\gamma}_{\lambda}^{+}(1)=u_{*} \\
\sigma\left(\tilde{\gamma}_{\lambda}^{+}(\tau)=\sigma_{+}\left(\tilde{\gamma}_{\lambda}^{+}(\tau)\right) \leq \sigma_{+}\left(\lambda \hat{u}_{0}(q)\right)=\sigma\left(\lambda \hat{u}_{0}(q)\right) .\right.
\end{gathered}
$$

It follows that $\tilde{\gamma}_{\lambda}^{+}$is a continuous path in $W$ which connects $\lambda \hat{u}_{0}(q)$ with $u_{*}$ and

$$
\sigma_{\mid \tilde{\gamma}_{\lambda}^{+}}<0
$$

In a similar fashion we produce $\tilde{\gamma}_{\lambda}^{-}$, a continuous path in $W$, which connects $-\lambda \hat{u}_{0}(q)$ with $v_{*}$ and

$$
\sigma_{\mid \tilde{\gamma}_{\lambda}^{-}}<0
$$


We concatenate paths $\tilde{\gamma}_{\lambda}^{-}, \tilde{\gamma}_{\lambda}, \tilde{\gamma}_{\lambda}^{+}$and generate $\gamma_{*} \in \Gamma$ s.t. $\sigma_{\mid \gamma_{*}}<0$, so $y_{0} \neq 0$ and $y_{0} \in\left[v_{*}, u_{*}\right] \cap \hat{C}^{1}(T)$ is a nodal solution of (1).

So, summarizing the situation for problem (1), we have the following multiplicity result.

Theorem 4.1. If hypotheses $\mathrm{H}(\mathrm{a})$ and $\mathrm{H}_{2}$ hold, then problem (1) has at least three nontrivial solutions

$$
u_{0} \in \operatorname{int} \hat{C}_{+}, v_{0} \in-\operatorname{int} \hat{C}_{+}, y_{0} \in\left[v_{0}, u_{0}\right] \text { nodal. }
$$

\section{A parametric problem with the scalar $p$-Laplacian}

In this section, we deal with the following parametric periodic problem driven by the scalar $p$-Laplacian.

$$
\left\{\begin{array}{l}
-\left(\left(\left|u^{\prime}(t)\right|\right)^{p-2} u^{\prime}(t)\right)^{\prime}=\lambda|u(t)|^{p-2} u(t)-f(t, u(t)) \text { a.e. on } T \\
u(0)=u(b), u^{\prime}(0)=u^{\prime}(b)
\end{array}\right.
$$

In this case $a(|x|) x=|x|^{p-2} x$ for all $x \in \mathbb{R}$. Thus in hypothesis $H(a)(i v), q=p$ and $\tilde{c}=1$, Our hypotheses on the perturbation $f(t, x)$ are rather general:

$H_{3}: f: T \times \mathbb{R} \rightarrow \mathbb{R}$ is a Carathéodory function s.t. $f(t, 0)=0$ for a.a. $t \in T$, and

(i) for every $\rho>0$ there exists $\alpha_{\rho} \in L^{\infty}(T)_{+}$s.t.

$$
|f(t, x)| \leq \alpha_{\rho}(t) \text { for a.a. } t \in T \text {, all }|x| \leq \rho ;
$$

(ii) $\lim _{x \rightarrow \pm \infty} \frac{f(t, x)}{|x|^{p-2} x}=+\infty$ uniformly for a.a. $t \in T$;

(iii) $\lim _{x \rightarrow 0} \frac{f(t, x)}{|x|^{p-2} x}=0$ uniformly for a.a. $t \in T$.

(iv) for every $\rho>0$ there exists $\xi_{\rho}>0$ s.t.

$$
\begin{aligned}
& -f(t,-\rho)-\xi_{\rho} \rho^{p-1} \leq-f(t,-x)-\xi_{\rho}|x|^{p-1} \\
& \quad \leq 0 \leq-f(t, x)+\xi_{\rho}|x|^{p-1} \leq-f(t, \rho)+\xi_{\rho} \rho^{p-1}
\end{aligned}
$$

for all $x \in[0, \rho]$, for all $t \in T$.

Remark 5.1. If $f(x)=|x|^{r-2} x$ with $p<r<+\infty$, then hypotheses $H_{3}$ are satisfied and (46) is a classical periodic equidiffusive $p$-logistic equation.

As a direct consequence of Theorem 4.1, we have the following multiplicity theorem.

Theorem 5.1. If hypotheses $H_{3}$ hold and $\lambda>\hat{\lambda}_{1}(p)$, then problem (46) has at least three nontrivial solutions

$$
u_{0} \in \operatorname{int} \hat{C}_{+}, v_{0} \in-\operatorname{int} \hat{C}_{+}, y_{0} \in\left[v_{0}, u_{0}\right] \text { nodal. }
$$

\section{Semilinear problems}

If in problem (46), $p=2$ (semilinear equation) and we improve the regularity of $f(t, \cdot)$, using Morse theory (critical groups), we can generate a second nodal solution for a total of four nontrivial solutions, all with sign information. The periodic problem under consideration is the following: 


$$
\left\{\begin{array}{l}
-u^{\prime \prime}(t)=\lambda u(t)-f(t . u(t)) \text { a.e. on } T \\
u(0)=u(b), u^{\prime}(0)=u^{\prime}(b)
\end{array}\right.
$$

We restrict ourselves to the semilinear problem because the stronger and more definitive results in Morse theory are obtained in the framework of Hilbert spaces for $C^{2}$-functionals (see hypotheses $H_{4}$ below).

The new stronger hypotheses on the perturbation $f(t, x)$ are rather general:

$H_{4}: f: T \times \mathbb{R} \rightarrow \mathbb{R}$ is a measurable function s.t. $f(t, 0)=0$ for a.a. $t \in T, f(t, \cdot) \in C^{1}(\mathbb{R})$ and

(i) there exists $\alpha_{\rho} \in L^{\infty}(T)_{+}, 2<r<+\infty$ s.t.

$$
\left|f_{x}^{\prime}(t, x)\right| \leq \alpha(t)\left(1+|x|^{r-2}\right) \text { for a.a. } t \in T
$$

(ii) $\lim _{x \rightarrow \pm \infty} \frac{f(t, x)}{x}=+\infty$ uniformly for a.a. $t \in T$;

(iii) $f_{x}^{\prime}(t, 0)=\lim _{x \rightarrow 0} \frac{f(t, x)}{x}=0$ uniformly for a.a. $t \in T$.

(iv) $f(t, x) x \geq 0$ for a.a. $t \in T$, all $x \in \mathbb{R}$.

Remark 6.1. Again the hypotheses incorporate the equidiffusive logistic equation (i.e. $f(t, x)=|x|^{r-2} x$ ).

The energy functional $\varphi_{\lambda}: W \rightarrow \mathbb{R}$ of problem (47) is defined by

$$
\varphi_{\lambda}(u)=\frac{1}{2}\|D u\|_{2}^{2}-\frac{\lambda}{2}\|u\|_{2}^{2}+\int_{0}^{b} F(t, u(t)) d t \text { for all } u \in W .
$$

Hypotheses $H_{4}$ imply that $\varphi_{\lambda} \in C^{2}(W)$ and

$$
\left\langle\varphi_{\lambda}(u) y, h\right\rangle=\int_{0}^{b}\left[y^{\prime}(t) h^{\prime}(t)-\lambda y(t) h(t)+f_{x}^{\prime}(t, u(t)) y(t) h(t)\right] d t \text { for all } u, y, h, \in W .
$$

Let $E\left(\hat{\lambda}_{k}\right)(2)$ be the eigenspace corresponding to the eigenvalue $\hat{\lambda}_{k}(2)$ of the periodic scalar Laplacian. Let $\bar{W}_{m}=\oplus_{k=0}^{m} E\left(\hat{\lambda}_{k}(2)\right)$ and $\widehat{W}_{m}=\bar{W}_{m}^{\perp}=$ $\overline{\oplus_{k \geq m+1} E\left(\hat{\lambda}_{k}(2)\right)}$. Then we have the following orthogonal direct sum decompositions

$$
W=\overline{\oplus_{k \geq 0} E\left(\hat{\lambda}_{k}\right)(2)}=\bar{W}_{m} \oplus \bar{W}_{m}^{\perp} .
$$

Proposition 6.1. If hypotheses $H_{4}$ hold and $\lambda>\hat{\lambda}_{1}(2)$, then $C_{k}\left(\varphi_{\lambda}, 0\right)=$ $\delta_{k, d_{m}} Z$ for all $k \geq 0$, where $d_{m}=\operatorname{dim} \bar{W}_{m}$ and $m \geq 1$ is such that $\lambda \in$ $\left(\hat{\lambda}_{m}(2), \hat{\lambda}_{m+1}(2)\right]$.

Proof. If $\lambda \in\left(\hat{\lambda}_{m}(2), \hat{\lambda}_{m+1}(2)\right)$, then $u=0$ is a nondegenerate critical point of $\varphi_{\lambda}$ with Morse index $d_{m}=\operatorname{dim} \bar{W}_{m}$ (see (48)) and (see [13], p. 155) this implies that

$$
C_{k}\left(\varphi_{\lambda}, 0\right)=\delta_{k, d_{m}} Z \text { for all } k \geq 0 .
$$

So, we need to consider the case $\lambda=\hat{\lambda}_{m+1}(2)$. By virtue of $H_{4}(i i i)$, given $\varepsilon>0$, we can find $\delta=\delta(\varepsilon)$ s.t.

$$
F(t, x) \leq \frac{\varepsilon}{2} x^{2} \text { for a.a. } t \in T, \quad \text { all }|x| \leq \delta .
$$


If $\bar{u} \in \bar{W}_{m}$, then we can find $\rho_{1}<0$ s.t.

$$
\|\bar{u}\| \leq \rho_{1} \Rightarrow|\bar{u}(t)| \leq \delta \text { for a.a. } t \in T
$$

Then for all $\bar{u} \in \bar{W}_{m}$ with $\|\bar{u}\| \leq \rho_{1}$ and any $\varepsilon \in\left(\hat{\lambda}_{m}(2), \hat{\lambda}_{m+1}(2)\right)$, taking into account also the variational characterization of $\hat{\lambda}_{m}(2)$ (see, for example, [13] (p.230)), we have

$$
\begin{aligned}
\varphi_{\lambda}(\bar{u}) & =\frac{1}{2}\left\|\bar{u}^{\prime}\right\|_{2}^{2}-\frac{\hat{\lambda}_{m+1}(2)}{2}\|\bar{u}\|_{2}^{2}+\int_{0}^{b} F(t, \bar{u}(t)) d t \\
& \leq \frac{\hat{\lambda}_{m}(2)-\hat{\lambda}_{m+1}(2)+\varepsilon}{2}\|\bar{u}\|_{2}^{2} \leq 0 .
\end{aligned}
$$

Next, let $\hat{u} \in \hat{W}_{m}$ and use $H_{4}(i v)$. We have

$$
\begin{aligned}
\varphi_{\lambda}(\hat{u}) & =\frac{1}{2}\left\|\hat{u}^{\prime}\right\|_{2}^{2}-\frac{\hat{\lambda}_{m+1}(2)}{2}\|\hat{u}\|_{2}^{2}+\int_{0}^{b} F(t, \hat{u}(t)) d t \\
& \geq \frac{1}{2}\left\|\hat{u}^{\prime}\right\|_{2}^{2}-\frac{\hat{\lambda}_{m+1}(2)}{2}\|\hat{u}\|_{2}^{2} \geq 0 .
\end{aligned}
$$

From (52) and (53) we see that $\varphi_{\lambda}$ has the local linking at the origin with respect to the orthogonal direct sum (49). So, from [20], we infer again that

$$
C_{k}\left(\varphi_{\lambda}, 0\right)=\delta_{k, d_{m}} Z \text { for all } k \geq 0 \text {. }
$$

Now we are ready for the multiplicity theorem for problem (47).

Theorem 6.1. If hypotheses $H_{4}$ hold and $\lambda>\hat{\lambda}_{1}(2)$, then problem (47) admits at least four nontrivial solutions

$$
u_{0} \in \operatorname{int} \hat{C}_{+}, v_{0} \in-\operatorname{int} \hat{C}_{+}, y_{0}, \hat{y} \in\left[v_{0}, u_{0}\right] \text { both nodal. }
$$

Proof. Note that the differentiability of $f(t, \cdot)$ and hypothesis $H_{4}(i)$ imply that for every $\rho>0$, we can find $\xi_{\rho}>0$ s.t. for a.a. $t \in T$

$$
x \rightarrow \xi_{\rho} x-f(t, x) \text { is nondecreasing on }[-\rho, \rho],
$$

hence hypotheses $H_{3}(i v)$ is satisfied (see Remark 3.1). Let $u_{0}, v_{0}$ and $y_{0}$ be the solutions obtained in Theorem 5.1, satisfying $v_{0} \leq y_{0} \leq u_{0}$. Thanks to Proposition 3.3, we may assume that $u_{0}$ and $v_{0}$ are extremal, that is $u_{0}=u_{*}$, $v_{0}=v_{*}$. Let $\rho=\max \left\{\left\|u_{0}\right\|_{\infty},\left\|v_{0}\right\|_{\infty}\right\}$ and let $\xi_{\rho}$ be as in (54). Then

$$
\begin{aligned}
-y_{0}^{\prime \prime}(t)+\xi_{\rho} y_{0}(t) & =\lambda y_{0}(t)-f\left(t, y_{0}(t)\right)+\xi_{\rho} y_{0}(t) \\
\leq \lambda u_{0}(t)-f\left(t, u_{0}(t)\right)+\xi_{\rho} u_{0}(t) & =-u_{0}^{\prime \prime}(t)+\xi_{\rho} u_{0}(t) \text { a.e. on } T,
\end{aligned}
$$

therefore $\left(u_{0}-y_{0}\right)^{\prime \prime}(t) \leq \xi_{\rho}\left(u_{0}-y_{0}\right)(t)$ a.e. on $T$, and (see [22])

$$
u_{0}-y_{0} \in \operatorname{int} \hat{C}_{+} \text {. }
$$

Similarly, we show that

$$
y_{0}-v_{0} \in \operatorname{int} \hat{C}_{+}
$$

so

$$
y_{0} \in \operatorname{int}_{\hat{C}^{1}(T)}\left[v_{0}, u_{0}\right] .
$$


Let $\sigma_{\lambda}$ be the truncated (at levels $u_{0}$ and $v_{0}$ ) and perturbed $C^{1}$-functional introduced in Section 4 and suppose that $K_{\sigma_{\lambda}}=\left\{0, u_{0}, v_{0}, y_{0}\right\}$. From Proposition 4.2 we know that $u_{0}$ and $v_{0}$ are local minimizers of $\sigma_{\lambda}$. Therefore

$$
C_{k}\left(\sigma_{\lambda}, u_{0}\right)=C_{k}\left(\sigma_{\lambda}, v_{0}\right)=\delta_{k, 0} Z \text { for all } k \geq 0 \text {. }
$$

From the proof of Proposition 4.3 we know that $y_{0}$ is a critical point of $\sigma_{\lambda}$ of mountain pass type. Therefore (see [13] (p. 176))

$$
C_{1}\left(\sigma_{\lambda}, y_{0}\right) \neq 0 \text {. }
$$

Since $\sigma_{\lambda \mid\left[v_{0}, u_{0}\right]}=\varphi_{\lambda \mid\left[v_{0}, u_{0}\right]}$, because of $(55)$, we have

$$
C_{k}\left(\sigma_{\lambda \mid \hat{C}_{+}^{1}(T)}, y_{0}\right)=C_{k}\left(\varphi_{\lambda \mid \hat{C}_{+}^{1}(T)}, y_{0}\right) \text { for all } k \geq 0,
$$

hence (see [14]) we deduce

$$
C_{k}\left(\sigma_{\lambda}, y_{0}\right)=C_{k}\left(\varphi_{\lambda}, y_{0}\right) \text { for all } k \geq 0,
$$

and using (57) we have also

$$
C_{1}\left(\varphi_{\lambda}, y_{0}\right) \neq 0
$$

Bearing in mind the regularity properties of $\varphi_{\lambda}$, from [13] (p. 177), (59) and (58), we infer that

$$
C_{k}\left(\sigma_{\lambda}, y_{0}\right)=C_{k}\left(\varphi_{\lambda}, y_{0}\right)=\delta_{k, 1} Z \text { for all } k \geq 0
$$

Since $u_{0} \in \operatorname{int} \hat{C}_{+}, v_{0} \in-\operatorname{int} \hat{C}_{+}$, arguing as for (58), we have

$$
C_{k}\left(\sigma_{\lambda}, 0\right)=C_{k}\left(\varphi_{\lambda}, 0\right) \text { for all } k \geq 0,
$$

so, from Proposition 6.1

$$
C_{k}\left(\sigma_{\lambda}, 0\right)=\delta_{k, d_{m}} Z \text { for all } k \geq 0 .
$$

The coercivity of $\sigma_{\lambda}$ (see (29)) leads to

$$
C_{k}\left(\sigma_{\lambda}, \infty\right)=\delta_{k, 0} Z \text { for all } k \geq 0 .
$$

From (56), (60), (61) and (62) and using the Morse relation with $t=-1$, we obtain

$$
2(-1)^{0}+(-1)^{1}+(-1)^{d_{m}}=(-1)^{0}, \text { that is }(-1)^{d_{m}}=0, \text { a contradiction. }
$$

So, there exists $\hat{y} \in K_{\sigma_{\lambda}}, \hat{y} \notin\left\{0, u_{0}, v_{0}, y_{0}\right\}$. From (30) we have $\hat{y} \in\left[v_{0}, u_{0}\right]$ and so $\hat{y}$ is a nodal solution of $(47)$ and $\hat{y} \in C^{1}(T)$. In fact as we did for $y_{0}$, we show that

$$
\hat{y} \in \operatorname{int}_{\hat{C}^{1}(T)}\left[v_{0}, u_{0}\right]
$$

Remark 6.2. We stress that the above multiplicity result is true for all $\lambda>$ $\hat{\lambda}_{1}(2)$, including the elements of the spectrum of the periodic scalar Laplacian. So, the result covers equations resonant at 0 . 


\section{Acknowledgements}

The authors have been partially supported by the Gruppo Nazionale per l'Analisi Matematica, la Probabilità e le loro Applicazioni (GNAMPA) of the Istituto Nazionale di Alta Matematica (INdAM)-project "Analisi non lineare e problemi ellittici".

\section{References}

[1] Aizicovici, S., Papageorgiou, N., Staicu, V,: Degree theory for operators of monotone type and nonlinear elliptic equations with inequality constraints. Mem. Am. Math. Soc. 196(915) (2008)

[2] Aizicovici, S., Papageorgiou, N., Staicu, V.: Nonlinear resonant periodic problems with concave terms. J. Math. Anal. Appl. 375, 342-364 (2011)

[3] Aizicovici, S., Papageorgiou N., Staicu, V.: Nodal and multiple solutions for nonlinear periodic problems with competing nonlinearities. Commun. Contemp. Math. 15, 263-293 (2013)

[4] Ambrosetti, A., Lupo, D.: On a class of nonlinear Dirichlet problems with multiple solutions. Nonlinear Anal. 8, 1145-1150 (1984)

[5] Ambrosetti, A., Mancini, G.: Sharp nonuniqueness results for some nonlinear problems. Nonlinear Anal. 3, 635-645 (1979)

[6] Ambrosetti, A., Rabinowitz, P.H.: Dual variational methods in critical point theory and applications. J. Funct. Anal. 24, 349-381 (1973)

[7] Brezis, H., Niremberg, L.: $H^{1}$ versus $C^{1}$ local minimizers. CRAS Paris. 317, 465-472 (1993)

[8] Diaz, J.I., Saá, J.E.: Existence et unicité de solutions positives pour certaines equations elliptiques quasilineaires. CRAS Paris 305, 521-524 (1987)

[9] Filippakis, M., Kristàly, A., Papageorgiou, N.S.: Existence of five nonzero solutions with exact sign for a p-laplacian equation. Discr. Continuous Dyn. Syst. 24, 405-440 (2009)

[10] Gasinski, L., Papageorgiou, N.S.: Nonlinear analysis, Ser. Math. Anal. Appl., vol. 9. Chapman and Hall/CRC Press, Boca Raton (2006)

[11] Kyritsi, S., Papageorgiou, N.S.: Multiple solutions for nonlinear periodic problems. Can. Math. Bull. 56, 366-377 (2013)

[12] Michael, E.: Continuous Selections II. Ann. Math. 64, 562-580 (1956)

[13] Motreanu, D., Motreanu, D., Papageorgiou, N.S.: Topological and variational methods with applications to nonlinear boundary value problems. Springer, New York (2014)

[14] Palais, R.: Homotopy theory of indefinite dimensional manifolds. Topology 5, 1$16(1966)$ 
[15] Papageorgiou, N.S., Kyritsi, S.: Handbook of applied analysis. Springer, New York (2009)

[16] Papageorgiou, E.H., Papageorgiou, N.S.: A multiplicity theorem for problems with the p-Laplacian J.Funct. Anal. 244, 63-77 (2007)

[17] Pucci, P., Serrin, J.: The maximum principle. Birkhäser, Basel (2007)

[18] Struwe, M.: A note on a result of Ambrosetti-Mancini. Ann. Mat. Pura Appl. 131, 107-115 (1982)

[19] Struwe, M.: Variational Methods. Applications to nonlinear partial differential equations and hamiltonian systems. Springer-Verlag, Berlin (2008)

[20] Su, J.: Semilinear elliptic boundary value problems with double resonance between two consecutive eigenvalues. Nonlinear Anal. 48, 881-895 (2002)

[21] Su, J., Li, H.: Multiplicity results for the two-point boundary value problems at resonance. Acta Math. Sci. 28, 152-162 (2006)

[22] Vázquez, J.L.: A strong maximum principle for some quasilinear elliptic equations. Appl. Math. Optim. 12, 191-202 (1984)

Giuseppina Barletta

DICEAM

Facoltà di Ingegneria

Università di Reggio Calabria

via Graziella

Loc. Feo di Vito

89122 Reggio Calabria

Italy

e-mail: giuseppina.barletta@unirc.it

Giuseppina D'Aguì

DICIEAMA

Università degli studi di Messina

c/da Di Dio, 98166 Messina

Italy

e-mail:dagui@unime.it

Nikolaos S. Papageorgiou

Department of Mathematics

National Technical University

Zografou Campus

Athens 15780

Greece

e-mail:npapg@math.ntua.gr

Received: 17 December 2014.

Accepted: 12 October 2015. 